\title{
Differentiation Between Sepsis Survivors and Sepsis Non-survivors Through Blood Serum Metabolomics: A Proton Nuclear Magnetic Resonance Spectroscopy (NMR) Study.
}

Naveen kumar M S

All India Institute of Medical Sciences

Virendra Kumar

All India Institute of Medical Sciences

Jagannathan N R

All India Institute of Medical Sciences

Sanjeev Sinha ( $\nabla$ drsanjeevsinha@gmail.com )

All India Institute of Medical Sciences

\section{Sujeet Mewar}

All India Institute of Medical Sciences

Pradeep Kumar

All India Institute of Medical Sciences

\section{Research Article}

Keywords: Sepsis, Nuclear Magnetic Resonance (NMR) spectroscopy, Blood Serum Metabolomics, Proton

Posted Date: April 16th, 2021

DOI: https://doi.org/10.21203/rs.3.rs-412947/v1

License: (c) (i) This work is licensed under a Creative Commons Attribution 4.0 International License.

Read Full License 


\section{Abstract}

Sepsis is a life-threatening organ dysfunction caused by a dysregulated host response to infection. Septic shock is a subset of sepsis with underlying circulatory cellular and metabolic abnormalities associated with higher mortality rates. However, a detailed understanding of sepsis is still limited. The present study reports the differences in the metabolic profile of serum samples of patients with sepsis compared to healthy controls using Nuclear Magnetic Resonance (NMR) spectroscopy. The study also compares the NMR metabolomics on day zero of admission among sepsis survivors (those who survived till day seven) and sepsis non-survivors (those who succumbed on day zero). Furthermore, the different metabolites in serum were analysed by univariate and multivariate analysis, ROC analysis, principal component analysis (PCA), partial least squares discriminant analysis (PLS-DA) and orthogonal partial least squares discriminant analysis (OPLS-DA) methods. Metabolites with VIP score $(>1 \cdot 0)$ were considered as potential biomarker/s to discriminate sepsis survivors from non- survivors at day zero. Data showed that phenylalanine was significantly higher in sepsis patients compared to healthy controls, whereas isoleucine, valine and histidine were significantly lower in sepsis patients compared to healthy controls. Also, non-survivors had higher serum levels of creatine, phosphocreatine, choline, betaine, tyrosine, histidine and phenylalanine concentrations than survivors. These findings suggest that the metabolic alterations at day zero may predict the survival of patients with sepsis. The significant differences seen in metabolites concentration of amino acids, phospholipids and creatine may be used as early prognostic markers to discriminate non-survivors from survivors of sepsis patients at day zero. Our findings indicate that the metabolite alterations are associated with the progression of the disease.

\section{Introduction}

Sepsis is one of the important global health problems. It is a life threatening condition leading to organ dysfunction as a result of host immune dysregulation in response to infection. The accurate estimation of incidence, mortality, standard diagnostic tests and therapeutics pertaining to sepsis remains a formidable challenge for researchers and healthcare providers ${ }^{1,2}$. A population-based analysis by Global Burden of Diseases, Injuries, and Risk Factors Study (GBD) 2017, estimated the global, regional, and national incidence of sepsis and mortality in about 195 countries for the years 1990 to 2017 . This study reported 48.9 million cases of sepsis and 11 million sepsis-related deaths, worldwide. The severely affected regions were those with lower socio-demographic Index (SDI) and the highest incidence was seen in Sub-Saharan Africa, Oceania, South Asia, East Asia, and Southeast Asia ${ }^{2}$. The definition of sepsis has been changing over the years, which reflects the changes in the understanding of sepsis and its underlying pathophysiology among the scientific community. Consequently, the third international consensus definitions of sepsis and septic shock (Sepsis-3) were published by a task force of the European Society of Intensive Care Medicine and the Society for Critical Care Medicine ${ }^{3}$. There is an increasing need of point of care technology with a fairly good diagnostic accuracy. The growing recognition that sepsis involves multiple tissues and organs with the consequent alteration in expression patterns of a variety of endogenous substances, has led to the description of a plethora of biomarkers of 
clinical utility, with varying degrees of specificity in diagnosing sepsis and validation in different clinical cohort ${ }^{4}$. In fact, the evaluation of existing biomarkers (TNF,IL-6,PCT) and the clinical scores such as Sequential Organ Failure Assessment score (SOFA) ${ }^{4}$ have been applied prognostically but their performance (sensitivity and specificity) has not been proven adequate for all cases ${ }^{5}$. Metabolomics is a "systematic study of unique metabolic fingerprints involving specific cellular processes" 6 . The metabolome represents the collection of small molecule chemicals in biological fluids (blood and urine), cells and tissues ${ }^{7}$. Metabolomics could supply key information and a better understanding of the biochemistry in system biology ${ }^{8,9}$. Proton $\left({ }^{1} \mathrm{H}\right)$ Nuclear Magnetic Resonance $\left({ }^{1} \mathrm{H}-\mathrm{NMR}\right)$ is one of the major analytical approaches of metabolomics, where a large number of metabolites in serum or urine or tissue is quantitatively analysed in a single step. It promises an immense potential for early diagnosis and for understanding the pathogenesis of the disease ${ }^{6}$.The literature on NMR spectroscopy based metabolomics approach to develop a personalized predictive model for septic patients has increased significantly in the last decade. The metabolomics reflects the molecular fingerprint of the deranged metabolic pathways, giving a novel insight into "metabolic theory of sepsis". In this study we hypothesize that there would be significant differences in the metabolic profiles of healthy controls compared to septic patients ${ }^{7,8}$. We investigated the discriminatory ability of metabolic fingerprints in early prediction of sepsis survivors and non-survivors within 24 hours and also describe the evolution of metabolic fingerprints in patients with sepsis using NMR spectroscopy based metabolomics.

\section{Materials And Methods}

\section{$2 \cdot 1$ Study design and participants}

This study was approved by the Institutional Ethics Committee of the All India Institute of Medical Sciences, New Delhi and was performed in accordance with the Declaration of Helsinki. A written informed consent was obtained from all patients or their relatives. This prospective observational study included patients admitted to the Department of Medicine, All India Institute of Medical Sciences, New Delhi from January 2018 to September 2019. Patients, who were diagnosed with sepsis within 24 hours of hospital admission and admitted to intensive care unit (ICU) were enrolled in the study. All patients were managed according to standard intensive care unit protocol and as per sepsis guidelines. Patients included in study were based on the following inclusion criteria: (One) patients of either gender and age more than eighteen years, and (Two) who met the sepsis criteria as defined by the Third International Consensus Definitions for Sepsis ${ }^{3,9}$. Patients were excluded if: (One) they were diagnosed with decompensated liver diseases and chronic kidney diseases; (Two) inherited or acquired immunodeficiency or on immunosuppressive therapy; (Three) pregnancy; (Four) post-operative sepsis; (Five) malignancies, and (Six) patients not willing to give consent for the study.

The following demographic data was recorded which included age, gender, presence or history of chronic diseases, reason for admission and cause of infection. To evaluate the severity of underlying diseases both Acute Physiological and Chronic Health Evaluation II (APACHE II) and Sequential Organ Failure 
Assessment score(SOFA) were calculated on the day of admission day Zero and on day Seven. Patients were followed up for a period of seven days. Those who succumbed within 24 hours of admission (day zero) were considered as non-survivors and those who survived till day seven were considered as survivors. Both survivors and non-survivors were chosen retrospectively once the outcomes were certain. Age and gender matched healthy volunteers were taken as controls.

\subsection{Sample Collection}

$5 \mathrm{ml}$ of venous blood was drawn at the time admission into ICU (day zero). Serum was obtained by collecting the blood into vacutainer and allowing the blood to clot for $45 \mathrm{~min}$ at room temperature. The samples were then centrifuged at $3000 \mathrm{rpm}$ for $15 \mathrm{~min}$, collected into one each $2 \mathrm{~mL}$ tube and stored at $80^{\circ} \mathrm{C}$ until used for NMR measurement.

\subsection{NMR spectroscopy and metabolite concentration profiling}

Before ${ }^{1} \mathrm{H}-\mathrm{NMR}$ analysis, the sample was processed for data acquisition. The serum was thawed and 200 $\mu \mathrm{L}$ aliquots were diluted with $340 \mu \mathrm{L}$ of $\mathrm{D}_{2} \mathrm{O} .30 \mu \mathrm{L}$ of TSP $(0.5 \mathrm{mM})$ and $30 \mu \mathrm{L}$ formate was added to serve as a chemical shift reference and concentration standard, respectively and transferred into a $5 \mathrm{~mm}$ NMR tube. Proton spectra of serum samples were carried out using a $700 \mathrm{MHz}$ spectrometer (Agilent, USA). One dimensional 1D CPMG with water pre-saturation was acquired at $25^{\circ} \mathrm{C}$ with following parameters: spectral width: $9124 \cdot 1 \mathrm{~Hz}$; scans: 64 ; relaxation delays: 70 s. The total spin-spin relaxation (TE) delay was kept at $15 \mathrm{~ms}$ to attenuate the broad NMR signals from macromolecules and to retain the signals of small metabolites, according to differences of T2 relaxation times from macromolecules and metabolites. Two dimensional (2D) total correlation spectroscopy (TOCSY) experiments were carried out for assignments of various metabolite resonances with the following parameters: $2 \mathrm{~K}$ data point, relaxation delay $=2 \cdot 5 \mathrm{~s}$; number of increments $=400(\mathrm{t} 1)$; number of scans $=16$; spin lock $=80 \mathrm{~ms}$.

\subsection{Statistical Analysis}

The spectroscopic data was processed using the Vnmrj 2.3A software (Agilent technologies). Metabolite concentrations were expressed as mean with SD or median with Interquartile range. Statistical analysis was carried out using SPSS software (SPSS Inc. Chicago, IL, USA) and MetaboAnalyst $4 \cdot 0$. Comparison between two groups was done using independent student t- test. A p-value less than 0.05 were considered significant. Receiver operating characteristics (ROC) curve analysis and multivariate analysis (orthogonal partial least squares discriminant analysis (OPLS-DA), variable importance to projection (VIP) score was also calculated. Additionally, a diagnostic model was constructed using the biomarker/s metabolites alone using linear discriminant analysis. The classification performance (sensitivity and specificity) of the OPLS-DA model and the area under the curve (AUC) of ROC were also calculated from the respective Monte-Carlo cross validation (MCCV) prediction.

\section{Results}




\subsection{Baseline characteristics of patients}

During the 20 months study period, a total of 146 patients with sepsis were admitted into ICU and 31 met the inclusion criteria. Out of 31 patients, 17 patients were non-survivors and 14 patients were survivors. 12 healthy controls were selected from the population after screening them for clinical and laboratory parameters. The baseline demographic, clinical and laboratory parameters of all patients are presented in Table 1. In this study, the majority of patients with sepsis were middle aged males with a mean age of 44.3 years $( \pm 12.8)$ and the ratio of male to female ratio was 3:1 in all the groups. At day zero, the average APACHE II and SOFA score in sepsis patients were $22 \cdot 2( \pm 13 \cdot 4)$ and $10 \cdot 4( \pm 5 \cdot 3)$, respectively. There was a statistically significant difference in APACHE II and SOFA score between the survivor and non-survivor groups $(p=0.001)$ 
Table 1

Baseline characteristics recorded at time of admission of all patients (both survivors and non-survivors)Demographic, clinical and laboratory variables

\begin{tabular}{|c|c|c|c|c|}
\hline \multirow[t]{2}{*}{ Characteristics } & \multicolumn{4}{|l|}{ Variables } \\
\hline & $\begin{array}{l}\text { All patients } \\
(\mathrm{n}=31)\end{array}$ & $\begin{array}{l}\text { Survivors } \\
(\mathrm{n}=14)\end{array}$ & $\begin{array}{l}\text { Non- } \\
\text { survivors } \\
(n=17)\end{array}$ & $\begin{array}{l}P \\
\text { value }\end{array}$ \\
\hline Male gender, n (\%) & $23(74 \cdot 19)$ & $10(71 \cdot 4)$ & $13(76 \cdot 4)$ & 0.5 \\
\hline Age, mean $( \pm S D)$ & $44 \cdot 3 \pm 12 \cdot 8$ & $46 \cdot 7 \pm 13 \cdot 7$ & $42 \pm 17 \cdot 3$ & $0 \cdot 32$ \\
\hline Septic shock, n (\%) & $24(77 \cdot 4)$ & $7(50)$ & $17(100)$ & 0.001 \\
\hline APACHE II on admission, median [min-max] & $27[2-38]$ & $8[2-14]$ & $34[24-38]$ & 0.001 \\
\hline SOFA score on admission, mean $( \pm S$ & $10 \cdot 5 \pm 5.3$ & $5 \cdot 2 \pm 2 \cdot 48$ & $14 \cdot 9 \pm 1 \cdot 9$ & 0.001 \\
\hline \multicolumn{5}{|l|}{ Focus of infection, $n(\%)$} \\
\hline Pulmonary & $17(54 \cdot 8)$ & $6(42 \cdot 8)$ & $11(64 \cdot 7)$ & \multirow[t]{6}{*}{$>0 \cdot 1$} \\
\hline Abdominal & $3(9 \cdot 6)$ & $1(7 \cdot 1)$ & $2(11 \cdot 7)$ & \\
\hline Urinary & $2(6 \cdot 4)$ & $1(7 \cdot 1)$ & $1(5 \cdot 8)$ & \\
\hline CNS & $4(12 \cdot 9)$ & $1(7 \cdot 1)$ & $3(17.6)$ & \\
\hline CVS & $2(6 \cdot 4)$ & $2(14 \cdot 2)$ & $0(0)$ & \\
\hline Skin and soft tissue & $3(9 \cdot 6)$ & $3(21 \cdot 4)$ & $0(0)$ & \\
\hline \multicolumn{5}{|l|}{ Chronic disease, n (\%) } \\
\hline $\begin{array}{l}\text { Post-tuberculosis sequelae and obstructive } \\
\text { lung disease }\end{array}$ & $6(19 \cdot 3)$ & $3(21 \cdot 4)$ & $3(17 \cdot 6)$ & 0.57 \\
\hline Diabetes & $9(28 \cdot 5)$ & $4(2 \cdot 7)$ & $5(29 \cdot 4)$ & 0.63 \\
\hline Hypertension & $6(18 \%)$ & $3(21 \cdot 4)$ & $3(17 \cdot 6)$ & 0.57 \\
\hline \multicolumn{5}{|l|}{ Clinical and laboratory parameters } \\
\hline Mean arterial pressure $(\mathrm{mmHg}), \operatorname{mean}( \pm \mathrm{SD})$ & $67( \pm 12 \cdot 8)$ & $75( \pm 8 \cdot 2)$ & $60( \pm 7 \cdot 2)$ & 0.004 \\
\hline Mechanical ventilation, $\mathrm{n}(\%)$ & $24(77)$ & $6(42)$ & $17(100)$ & 0.001 \\
\hline
\end{tabular}

Data are expressed as medians [min-max] or with frequencies and percentages. $p$ value is statistically significant when $<0.05$. APACHE II = acute physiology and chronic health evaluation II, SOFA = sequential organ failure assessment, $\mathrm{PCT}$ procalcitonin, $\mathrm{CRP} \mathrm{C}$ reactive protein, $\mathrm{PaO}_{2}$ partial pressure of arterial oxygen, $\mathrm{PaCO}_{2}$ partial pressure of arterial carbon dioxide, $\mathrm{SpO}_{2}$ oxygen saturation, $\mathrm{FiO}_{2}$ percentage of inspired oxygen, CNS central nervous system, CVS cardiovascular system, SD standard deviation, and IQR interquartile range. 


\begin{tabular}{|c|c|c|c|c|}
\hline Characteristics & Variables & & & \\
\hline $\mathrm{SpO}_{2}(\mathrm{mmHg})$, median[min-max] & $90[60-98]$ & $95[89-98]$ & $88[66-99]$ & 0.007 \\
\hline $\mathrm{pH}$ at admission, median[min-max] & $\begin{array}{l}7 \cdot 10[6 \cdot 90- \\
7 \cdot 54]\end{array}$ & $\begin{array}{l}7 \cdot 32[7 \cdot 16- \\
7 \cdot 54]\end{array}$ & $\begin{array}{l}7 \cdot 04[6 \cdot 9- \\
7 \cdot 10]\end{array}$ & 0.001 \\
\hline $\mathrm{PaO}_{2}(\mathrm{mmHg})$, median[min-max] & $\begin{array}{l}114[51- \\
196]\end{array}$ & $80[51-196]$ & $\begin{array}{l}122[64- \\
187]\end{array}$ & 0.96 \\
\hline $\mathrm{PaCO}_{2}(\mathrm{mmHg})$, median[min-max] & $22[12-94]$ & $24[15-46]$ & $22[12-94]$ & 0.75 \\
\hline $\mathrm{PaO}_{2} / \mathrm{FiO}_{2}$, mean $( \pm \mathrm{SD})$ & $211( \pm 102)$ & $280( \pm 97)$ & $152( \pm 68)$ & 0.002 \\
\hline Bicarbonate (mmol/l, median[min-max]) & $17[6-30]$ & $19[9-30]$ & $14[6-25]$ & 0.008 \\
\hline Hemoglobin(g/dL), median[min-max] & $9 \cdot 0[5 \cdot 2-13]$ & $10 \cdot 2[7 \cdot 5-13]$ & $7 \cdot 6[5 \cdot 2-12]$ & 0.001 \\
\hline Leukocyte $\left(x 10^{9} / \mathrm{I}\right)$, median[min-max] & $15 \cdot 6[1 \cdot 0-30]$ & $11[1 \cdot 1-25 \cdot 3]$ & $\begin{array}{l}16 \cdot 1[1 \cdot 0- \\
30]\end{array}$ & $0 \cdot 2$ \\
\hline Platelets (x $\left.10^{9} / \mathrm{l}\right)$, median[min-max] & $70[2-452]$ & $86[20-452]$ & $60[2-128]$ & 0.02 \\
\hline $\begin{array}{l}\text { Random blood glucose(mg/dl), median[min- } \\
\text { max]; }\end{array}$ & $\begin{array}{l}134[11- \\
288]\end{array}$ & $\begin{array}{l}\text { 131[11- } \\
199]\end{array}$ & $\begin{array}{l}144[12- \\
288]\end{array}$ & $0 \cdot 24$ \\
\hline Bilirubin(mg/dl), median[min-max] & $1[0 \cdot 8-1 \cdot 6]$ & $\begin{array}{l}0 \cdot 85[\cdot 72- \\
1 \cdot 0]\end{array}$ & $1 \cdot 4[1-2 \cdot 2]$ & 0.006 \\
\hline $\operatorname{Albumin}(\mathrm{g} / \mathrm{L}), \operatorname{mean}( \pm \mathrm{SD})$ & $2 \cdot 42( \pm 0 \cdot 8)$ & $2 \cdot 56( \pm 1 \cdot 1)$ & $2 \cdot 28(0 \cdot 46)$ & $0 \cdot 1$ \\
\hline РCT (ng/ml), median[min-max]; & $\begin{array}{l}15[0 \cdot 14- \\
200]\end{array}$ & $5[0 \cdot 14-21]$ & $27[2-200]$ & 0.002 \\
\hline Lactate(mmol/l), median[min-max]; & $4[1 \cdot 2-8]$ & $2[1 \cdot 2-5]$ & $3[3-8]$ & 0.001 \\
\hline Creatinine(mg/dl), median[min-max]; & $1 \cdot 3[0 \cdot 4-7]$ & $1 \cdot 1[0 \cdot 4-1 \cdot 8]$ & $\begin{array}{l}2 \cdot 2[0 \cdot 5- \\
1 \cdot 8]\end{array}$ & 0.005 \\
\hline $\operatorname{Urea}(\mathrm{mg} / \mathrm{dl})$, median[min-max]; & $77[15-259]$ & $44[15-181]$ & $93[29-259]$ & 0.008 \\
\hline $\mathrm{CRP}(\mathrm{mg} / \mathrm{dL}), \operatorname{mean}( \pm \mathrm{SD})$ & $100( \pm 31)$ & $81 \cdot 2( \pm 24)$ & $113( \pm 35)$ & $0 \cdot 2$ \\
\hline \multicolumn{5}{|c|}{$\begin{array}{l}\text { Data are expressed as medians [min-max] or with frequencies and percentages. } \mathrm{p} \text { value is statistically } \\
\text { significant when }<0 \cdot 05 \text {. APACHE II = acute physiology and chronic health evaluation } \mathrm{II}, \mathrm{SOFA}= \\
\text { sequential organ failure assessment, } \mathrm{PCT} \text { procalcitonin, } \mathrm{CRP} \mathrm{C} \text { reactive protein, } \mathrm{PaO}_{2} \text { partial pressure } \\
\text { of arterial oxygen, } \mathrm{PaCO} \mathrm{CO}_{2} \text { partial pressure of arterial carbon dioxide, } \mathrm{SpO}_{2} \text { oxygen saturation, } \mathrm{FiO}_{2} \\
\text { percentage of inspired oxygen, CNS central nervous system, } \mathrm{CVS} \text { cardiovascular system, } \mathrm{SD} \text { standard } \\
\text { deviation, and IQR interquartile range. }\end{array}$} \\
\hline
\end{tabular}

\subsection{Serum metabolites in Sepsis vs Healthy control}


A total of 43 serum samples were collected; 14 samples from survivors, 17 from non-survivors within 24 hours of admission and 12 healthy controls. In each sample, the metabolites were identified using their chemical shifts values and the concentrations were measured. Table 2 compares the serum concentration of metabolites between patients with sepsis and healthy controls. There are four metabolites, which show significant difference between the two groups. Compared with the healthy controls, the sepsis patients had significantly higher serum concentration of phenylalanine and lower concentrations of isoleucine, valine and histidine. OPLS-DA score plots were used to compare the differences in metabolites in sepsis and healthy control groups.. In Fig. 1, the OPLS-DA score plot shows a clear discrimination between sepsis patients and healthy controls. Figure 1 also shows the VIP score plot of metabolites in sepsis and healthy controls in descending order of importance. Among the metabolites (in descending order of importance), phenylalanine isoleucine, histidine and valine were useful in differentiating sepsis from controls. 
Table 2

Comparison of serum metabolites concentration among patients with sepsis and healthy controls.

\begin{tabular}{|c|c|c|c|c|}
\hline \multirow[t]{3}{*}{ Metabolites } & \multirow{3}{*}{$\begin{array}{l}\text { Chemical shift value } \\
\text { (ppm) }\end{array}$} & \multicolumn{2}{|c|}{ Metabolites concentration $\mathrm{mM}$} & \multirow[t]{3}{*}{ p-value } \\
\hline & & Patients with sepsis & Healthy control & \\
\hline & & $\mathrm{n}=\mathbf{3 1}$ & $n=12$ & \\
\hline Isoleucine & $0.94(d)$ & $0.21(0.17-.26)$ & $0.31(0.27-0.38)$ & 0.001 \\
\hline Valine & $1.04(d)$ & $0.27(0.20-.36)$ & $0.36(0.30-0.39)$ & 0.009 \\
\hline Alanine & $1.48(d)$ & $0.71(0.33)$ & $0.80(.23)$ & 0.3 \\
\hline Acetate & $1.91(\mathrm{~s})$ & $0.13(0.07-0.159)$ & $0.10(0.08-0.18)$ & 0.6 \\
\hline Pyruvate & $2.37(s)$ & $0.09(0.05)$ & $0.09(0.04)$ & 0.8 \\
\hline Citrate & $2.56(d)$ & $0.22(0.09)$ & $0.26(0.06)$ & 0.2 \\
\hline Creatine & $3.03(\mathrm{~s})$ & $0.28(0.16-.38)$ & $0.18(0.16-0.22)$ & 0.06 \\
\hline Phosphocreatine & $3.04(s)$ & $0.18(0.12-.30)$ & $0.15(0.13-0.23)$ & 0.65 \\
\hline Choline & $3.21(s)$ & $0.12(0.10-0.18)$ & $0.16(0.12-0.20)$ & 0.06 \\
\hline Betaine & $3.26(s)$ & $0.25(0.15-0.47)$ & $0.26(0.22-0.29)$ & 0.78 \\
\hline Glycine & $3.56(\mathrm{~s})$ & $0.18(0.12-0.26)$ & $0.26(0.21-0.27)$ & 0.2 \\
\hline Tyrosine & $6.88(d)$ & $0.12(0.07)$ & $0.09(0.026)$ & 0.18 \\
\hline Histidine & $7.06(\mathrm{~s})$ & $0.05(0.028)$ & $0.07(.014)$ & 0.03 \\
\hline Phenylalanine & $7.33(\mathrm{t})$ & $0.16(.08)$ & $0.09(0.03)$ & $<0.01$ \\
\hline \multicolumn{5}{|c|}{ Values are expressed as Mean(SD) or Median(quartile1- quartile3) } \\
\hline \multicolumn{5}{|c|}{ Serum values are presented as $\mathrm{mmol} / \mathrm{L}$. A p-value of less than 0.05 is statistically significant. } \\
\hline
\end{tabular}

\subsection{Sepsis survivors and non-survivors}

Table 3 shows the metabolites including amino acids (tyrosine, histidine and phenylalanine), products of muscle metabolism (creatine and phosphocreatine), and phospholipids (choline, and betaine) were found to be increased in the non-survivor group and were statistically significant. Figure 2 shows the OPLS-DA plot showing a clear discrimination between sepsis non-survivors and survivors. Figure 2 also shows the VIP plot score of metabolites in sepsis non-survivors and survivors in descending order of importance. Among the metabolites, phosphocreatine, tyrosine, choline, creatine, histidine and phenylalanine in descending order of importance were useful in differentiating sepsis non-survivors from survivors. 
Table 3

Comparison of serum metabolites concentration between survivors and non-survivor sepsis patients

\section{Chemical shift value (ppm) Metabolites concentration $\mathrm{mM}$}

Sepsis survivor Sepsis non- survivor

\begin{tabular}{|lllll|}
\hline Metabolites & & $\mathrm{n}=\mathbf{1 4}$ & $\mathrm{n}=17$ & $\mathrm{p}$-value \\
\hline Isoleucine & $0.94(\mathrm{~d})$ & $0.20(0.17-0.27)$ & $0.21(0.18-.24)$ & 0.73 \\
\hline Valine & $1.04(\mathrm{~d})$ & $0.24(0.18-0.41)$ & $0.28(0.232-.37)$ & 0.34 \\
\hline Alanine & $1.48(\mathrm{~d})$ & $0.58(0.51-.69)$ & $0.65(0.46-1.08)$ & 0.24 \\
\hline Acetate & $1.91(\mathrm{~s})$ & $0.11(0.07)$ & $0.14(0.06)$ & 0.23 \\
\hline Pyruvate & $2.37(\mathrm{~s})$ & $0.07(0.03)$ & $0.11(0.06)$ & 0.08 \\
\hline Citrate & $2.56(\mathrm{~d})$ & $0.19(0.05)$ & $0.25(0.11)$ & 0.06 \\
\hline Creatine & $3.03(\mathrm{~s})$ & $0.20(0.13-0.26)$ & $0.37(0.29-.45)$ & 0.001 \\
\hline Phosphocreatine & $3.04(\mathrm{~s})$ & $0.13(0.12-0.15)$ & $0.30(0.19-.49)$ & 0.0002 \\
\hline Choline & $3.21(\mathrm{~s})$ & $0.11(0.1-0.12)$ & $0.15(0.11-0.19)$ & 0.011 \\
\hline Betaine & $3.26(\mathrm{~s})$ & $0.21(0.12)$ & $0.40(0.22)$ & 0.01 \\
\hline Glycine & $3.56(\mathrm{~s})$ & $0.19(0.07)$ & $0.24(0.13)$ & 0.17 \\
\hline Tyrosine & $6.88(\mathrm{~d})$ & $0.08(0.06-0.09)$ & $0.14(0.08-0.2)$ & 0.01 \\
\hline Histidine & $7.06(\mathrm{~s})$ & $0.04(0.01)$ & $0.06(0.03)$ & 0.02 \\
\hline Phenylalanine & $7.33(\mathrm{t})$ & $0.12(0.4)$ & $0.19(0.10)$ & 0.04 \\
\hline Values are expressed as Mean(SD) or Median(quartile1- quartile3) & & \\
\hline Serum values are presented as mmol/L. A p-value of less than 0.05 is statistically significant. & \\
\hline
\end{tabular}

\subsection{Metabolic pathway analysis}

In addition, a metabolic pathway analysis was performed based on the identified biomarkers in serum using Metabo Analyst 4.0 software (Fig. 3) to identify the relevant metabolic pathways related to sepsis survivor and non-survivor patients, respectively and compared them with that of healthy controls. The impact value of significant metabolic pathways calculated from pathway topology analysis above $0 \cdot 1$ was screened out as potential target pathway. Based on the impact value, there were five potential target metabolic pathways identified which were related to sepsis patients compared to healthy controls. In 
patients with sepsis, the potential target metabolic pathways were phenylalanine, tyrosine, tryptophan biosynthesis, histidine metabolism, valine, leucine and isoleucine degradation and glycerophospholipid metabolism.

\section{Discussion}

\section{4·1 Demographic Characteristics: Comparison with global patient profile}

In this study, the mean age of the sepsis group was $44 \cdot 3( \pm 12.5)$ years when compared to the mean age of 60 years in the previous studies reported in the literature ${ }^{7,10}$. Male had more predispositions to sepsis, which is consistent with the earlier studies, which has been ascribed to variety of factors including differing inherent predisposition to infection and hormonal regulation of cytokine responses (11-13). The age and the percentage of male were matched in both the groups of survivor and non-survivors, thus avoiding potential confounding factors. The source of infection in majority of the cases was pulmonary (64\%) followed by CNS infections (9\%). $95 \%$ of patients had radiological evidence of infection whereas microbiological cultures was positive in $33 \%$ of the recruited cases with a blood culture positivity rate of $9 \%$.

Mean SOFA scores were $5.2( \pm 2.4)$ and $14.9( \pm 1.9)$ in survivor and non-survivor groups, respectively. APACHE had a perfect linear correlation $(\mathrm{r} 2=.92)$ with SOFA as a predictor of outcome of sepsis. Sepsis survivors had a mean APACHE of $8 \cdot 1 \pm 3.57$ ( $8 \%$ predicted mortality) while it was $34.5 \pm 2 \cdot 82$ for nonsurvivors (73\% mortality).

\subsection{Metabolomics profile of sepsis and healthy controls}

Metabolic changes in sepsis are highly complex and vary between individuals ${ }^{14}$. In the present study, four amino acids namely, phenylalanine, histidine, valine and isoleucine were found to differentiate between sepsis from healthy controls. Phenylalanine increased to a significant level in sepsis patients when compared to healthy controls. Muscle breakdown coupled with reduced conversion of phenylalanine to tyrosine in liver in sepsis contributes to higher levels of phenylalanine. This observation is consistent with the finding of previous studies ${ }^{15,16}$. Phenylalanine is also markedly increased in HIV ${ }^{17}$, trauma, burns, malignancy ${ }^{18}$ and SIRS $^{19}$. These reflect that phenylalanine elevation is a sign of immune activation rather than infection ${ }^{20}$. Phenylalanine is converted into fumarate, which is then shunted into Kreb's cycle for energy production under severe anorexic condition. Increased level of phenylalanine was associated with poor outcome in sepsis patients ${ }^{21}$.

Histidine concentration was lower in sepsis group when compared to controls. The trend of decreased histidine in sepsis is consistent with the results of the study by Jauria et al. ${ }^{22}$. Histidine has antioxidant and anti-inflammatory activity and low plasma histidine is associated with oxidative stress, inflammation and mortality in CKD (Chronic Kidney Disease) patient ${ }^{23}$. Histidine rich glycoprotein, a key regulator in immunity and vascular biology ${ }^{24}$ is decreased in sepsis and is being exploited as a potential prognostic 
marker in sepsis ${ }^{25}$.Concentration of branched chain amino acids (BCAA) namely isoleucine and valine were significantly higher in sepsis patients than in healthy controls, which agrees with the study by Mickwickz et al. ${ }^{26,16}$. Increased oxidation of BCAA by skeletal muscle coupled with conversion into glutamine decreases the BCAA levels in sepsis ${ }^{27}$.

Levels of choline was seen to be decreased significantly in sepsis patients compared to healthy controls and it increased in sepsis non-survivors compared to survivors. Choline is an endogenous molecule that has antioxidant, anti-apoptotic properties and attenuates the pro inflammatory cytokines (IL-6,IL-1ß,TNF邓) and protects the cell membrane from oxidant injury during various pathological conditions including sepsis $^{28}$. Choline supplementation in sepsis has improved outcomes in mammalian model ${ }^{29}$. Among the metabolites, isoleucine has the highest diagnostic performance with AUC of $0.898(0.781-0.973)$ followed by phenylalanine $0.858(0.683-0.976)$ in predicting sepsis (Fig. 4). These results are in concordance with the study of Zhicheng et al., where phenylalanine had a AUC of 0.84 in differentiating sepsis from control ${ }^{16}$.

\subsection{Metabolomics profile of Sepsis survivor from non- survivors}

In the present study, the concentration of phosphocreatine and creatine was significantly higher among sepsis non-survivors. Phosphocreatine-creatine system acts as a reservoir of energy which is available instantaneously. The ATP (Adenosine Tri Phosphate) combines with creatine to form high energy Phosphocreatine catalysed by Creatine kinase. In hypermetabolic state, the reaction reverses to yield ATP and creatine. This system is of particular importance in skeletal muscle and cardiac myocytes for rapid replenishment of ATP. Sepsis profoundly affects mitochondrial functions resulting in instability of membrane potential. There is autophagy induction, suppression of biogenesis inciting myocyte death with a leak of cytoplasmic creatine and phosphocreatine into serum ${ }^{30}$. Multiple studies have confirmed the loss of phosphocreatine in muscle fibres of sepsis patient with increased mortality ${ }^{31,32}$.

Further, the serum levels of tyrosine, histidine and phenylalanine were found to be significantly increased in the sepsis non-survivor group. The AUC (Fig. 5 ) is highest for phosphocreatine (0.89) followed by creatine $(0.83)$, choline $(0.76)$ and tyrosine $(0.75)$ for differentiating sepsis non-survivors from survivors. Elevation of phenylalanine in non-survivors has been consistently observed in many studies in the past ${ }^{33,16}$. Level of histidine and tyrosine in non-survivors are lower in non-survivors than survivors in the above studies. In the present study, the higher levels of these metabolites in non-survivors may be due to the inability to metabolise the amino acids reflecting the metabolic shutdown in patients who did not survive. A similar trend was seen with all the metabolites listed in Table 3 including choline. This is similar to the observation by Hirose et $\mathrm{al}^{33}$, i.e., the trends of metabolites in serum of an individual with sepsis varies greatly with time and severity of sepsis.

\section{Conclusion}


Thus the results of the present study indicated that NMR determined metabolite profiling may serve as a promising tool for the diagnosis and prognosis of sepsis. Integration of NMR metabolomics with the conventional sepsis biomarkers and SOFA may serve as a better indicator for early identification of patients who need aggressive therapy. In the evolving era of precision medicine, addition of Quantitative metabolomics can identify the dysregulated biochemical pathways in sepsis, which can be therapeutically targeted.

\section{Declarations}

\section{Acknowledgements:}

The authors thank the Department of Science and Technology (DST) for funds to buy a $700 \mathrm{MHz}$ NMR spectrometer (IR/SO/LU-05/2007/AllMS). NRJ thanks the Scientific and Research Board (SERB) of DST for the award of $\mathrm{J} \cdot \mathrm{C} \cdot$ Bose Fellowship.

\section{References}

1. Thompson K, Venkatesh B, Finfer S. Sepsis and septic shock: current approaches to management. Internal Medicine Journal. 2019;49(2):160-70.

2. Rudd KE, Johnson SC, Agesa KM, et al. Global, regional, and national sepsis incidence and mortality, 1990-2017: analysis for the Global Burden of Disease Study. The Lancet 2020 Jan 18;395(10219):200-11.

3. Singer M, Deutschman CS, Seymour CW, et al. The Third International Consensus Definitions for Sepsis and Septic Shock (Sepsis-3). JAMA 2016 Feb 23;315(8):801-10.

4. Reinhart K, Bauer M, Riedemann NC, Hartog CS. New approaches to sepsis: molecular diagnostics and biomarkers. Clin Microbiol Rev. 2012 Oct;25(4):609-34.

5. van Engelen TSR, Wiersinga WJ, Scicluna BP, van der Poll T. Biomarkers in Sepsis. Crit Care Clin. 2018 Jan;34(1):139-52.

6. Gowda GAN, Zhang S, Gu H, Asiago V, Shanaiah N, Raftery D. Metabolomics-based methods for early disease diagnostics. Expert Rev Mol Diagn. 2008 Sep;8(5):617-33.

7. Garcia-Simon M, Morales JM, Modesto-Alapont V, et al. Prognosis Biomarkers of Severe Sepsis and Septic Shock by 1H NMR Urine Metabolomics in the Intensive Care Unit. PLOS ONE. 2015 Nov 13;10(11):e0140993.

8. Liu Z, Triba MN, Amathieu R, et al. Nuclear magnetic resonance-based serum metabolomic analysis reveals different disease evolution profiles between septic shock survivors and non-survivors. Critical Care. 2019 May 14;23(1):169.

9. Wentowski C, Mewada N, Nielsen ND. Sepsis in 2018: a review. Anaesthesia \& Intensive Care Medicine. 2019 Jan 1;20(1):6-13. 
10. Jaurila H, Koivukangas V, Koskela M, et al. 1H NMR Based Metabolomics in Human Sepsis and Healthy Serum. Metabolites [Internet]. 2020 Feb 15 [cited 2020 Apr 14]

11. Rhee C, Klompas M. Sepsis trends: increasing incidence and decreasing mortality, or changing denominator? Journal of Thoracic Disease. 2020 Aug 1;12(S1):S89-S100

12. Ferrer R, Artigas A, Suarez D ,et al. Effectiveness of treatments for severe sepsis: a prospective, multicenter, observational study. Am J Respir Crit Care Med. 2009 Nov 1;180(9):861-6.

13. Naqvi IH, Mahmood K, Ziaullaha S, Kashif SM, Sharif A. Better prognostic marker in ICU - APACHE II, SOFA or SAP II! Pak J Med Sci. 2016;32(5):1146-51.

14. Van Wyngene L, Vandewalle J, Libert C. Reprogramming of basic metabolic pathways in microbial sepsis: therapeutic targets at last? EMBO Mol Med. 2018;10(8).

15. Liu Z, Triba MN, Amathieu R, et al. Nuclear magnetic resonance-based serum metabolomic analysis reveals different disease evolution profiles between septic shock survivors and non-survivors. Crit Care. 2019 14;23(1):169.

16. Su L, Li H, Xie A, et al. Dynamic Changes in Amino Acid Concentration Profiles in Patients with Sepsis. Boudko D, editor. PLOS ONE. 2015 Apr 7;10(4):e0121933.

17. Ollenschläger G, Jansen S, Schindler J, Rasokat H, Schrappe-Bächer M, Roth E. Plasma amino acid pattern of patients with HIV infection. Clin Chem. 1988 Sep;34(9):1787-9.

18. Watanabe A, Higashi T, Sakata T, Nagashima H. Serum amino acid levels in patients with hepatocellular carcinoma. Cancer. 1984 Nov 1;54(9):1875-82.

19. Druml W, Heinzel G, Kleinberger G. Amino acid kinetics in patients with sepsis. The American Journal of Clinical Nutrition. 2001 May 1;73(5):908-13.

20. Geisler S, Gostner JM, Becker K, Ueberall F, Fuchs D. Immune activation and inflammation increase the plasma phenylalanine-to-tyrosine ratio. Pteridines. 2013 Jun 1;24(1):27-31.

21. Cooney RN, Kimball SR, Vary TC. Regulation of skeletal muscle protein turnover during sepsis: mechanisms and mediators. Shock. 1997 Jan;7(1):1-16.

22. Jaurila H, Koivukangas V, Koskela M, et al. 1H NMR Based Metabolomics in Human Sepsis and Healthy Serum. Metabolites. 2020 Feb 15;10(2):70.

23. Watanabe M, Suliman ME, Qureshi AR, et al. Consequences of low plasma histidine in chronic kidney disease patients: associations with inflammation, oxidative stress, and mortality. The American Journal of Clinical Nutrition. 2008 Jun 1;87(6):1860-6.

24. Poon IKH, Patel KK, Davis DS, Parish CR, Hulett MD. Histidine-rich glycoprotein: the Swiss Army knife of mammalian plasma. Blood. 2011 Feb 17;117(7):2093-101.

25. Kuroda K, Wake H, Mori S, Hinotsu S, Nishibori M, Morimatsu H. Decrease in Histidine-Rich Glycoprotein as a Novel Biomarker to Predict Sepsis Among Systemic Inflammatory Response Syndrome: Critical Care Medicine. 2018 Apr;46(4):570-6.

26. Mickiewicz B, Duggan GE, Winston BW, Doig C, Kubes P, Vogel HJ. Metabolic Profiling of Serum Samples by $1 \mathrm{H}$ Nuclear Magnetic Resonance Spectroscopy as a Potential Diagnostic Approach for 
Septic Shock*: Critical Care Medicine. 2014 May;42(5):1140-9.

27. Bower RH, Vallgren S, Lafrance R, et al. Branched Chain Amino Acid-Enriched Solutions in the Septic Patient: A Randomized, Prospective Trial. Annals of Surgery. 1986 Jan;203(1):13-20.

28. Ilcol YO, Yilmaz Z, Ulus IH. Endotoxin alters Serum-Free Choline and Phospholipid-Bound Choline Concentrations, And Choline Administration Attenuates Endotoxin-Induced Organ Injury in Dogs: Shock. 2005 Sep;24(3):288-93.

29. Al-Humadi A, Al-Humadi H, Liapi C. Novel insight on the impact of choline-deficiency in sepsis. Ann Res Hosp. 2019 Jun;3:12-12.

30. Park DW, Zmijewski JW. Mitochondrial Dysfunction and Immune Cell Metabolism in Sepsis. Infect Chemother. 2017 Mar;49(1):10-21.

31. Gilles RJ, D'orio V, Ciancabilla F, Carlier PG. In vivo 31P nuclear magnetic resonance spectroscopy of skeletal muscle energetics in endotoxemic rats: A prospective, randomized study. Critical Care Medicine. 1994 Mar;22(3):499-505.

32. Hotchkiss RS. Reevaluation of the Role of Cellular Hypoxia and Bioenergetic Failure in Sepsis. JAMA. 1992 Mar 18;267(11):1503.

33. Hirose $\mathrm{T}$, Shimizu $\mathrm{K}$, Ogura $\mathrm{H}$, et al. Altered balance of the aminogram in patients with sepsis - The relation to mortality. Clinical Nutrition. 2014 Feb;33(1):179-82.

\section{Figures}



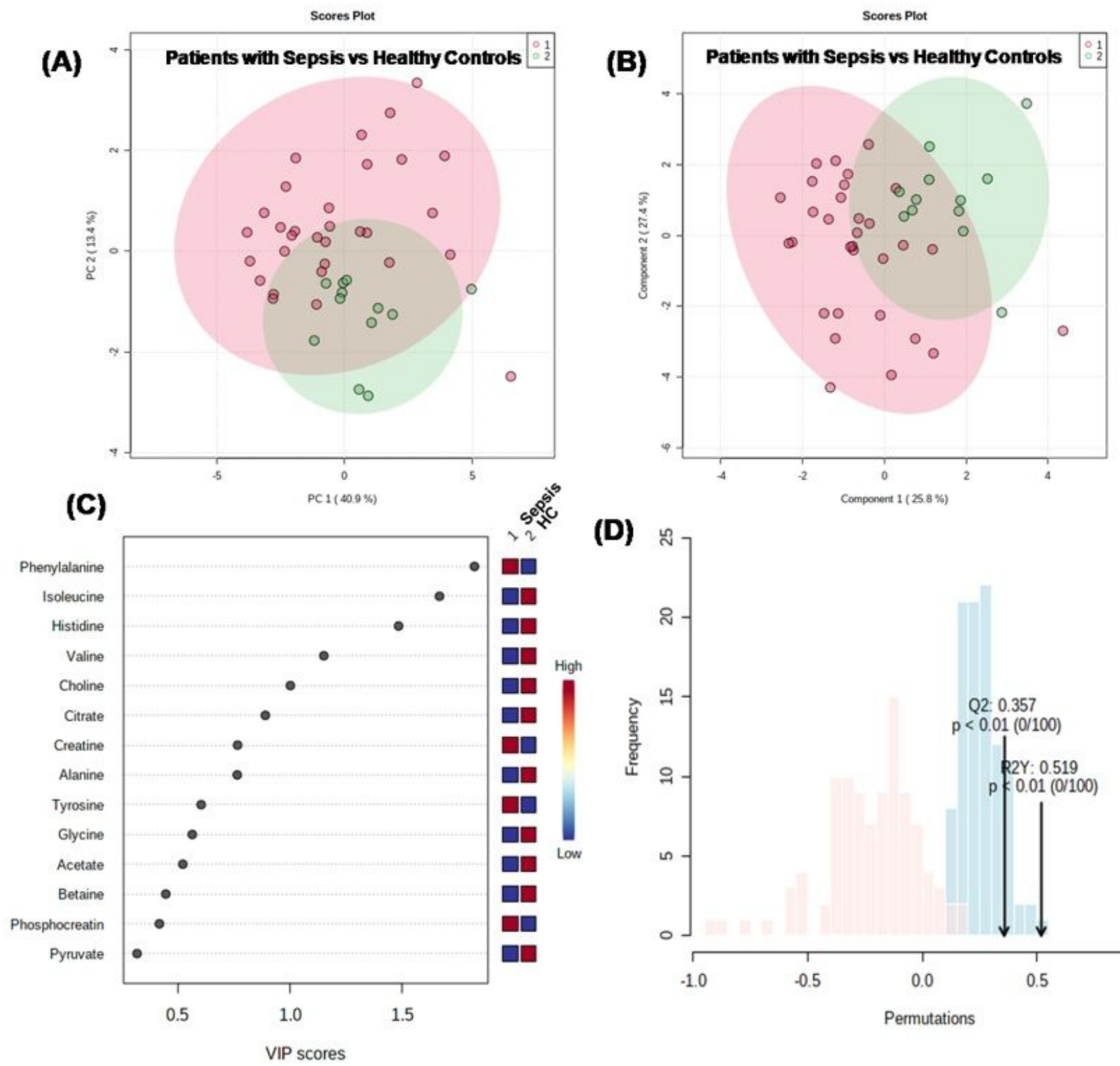

(D)

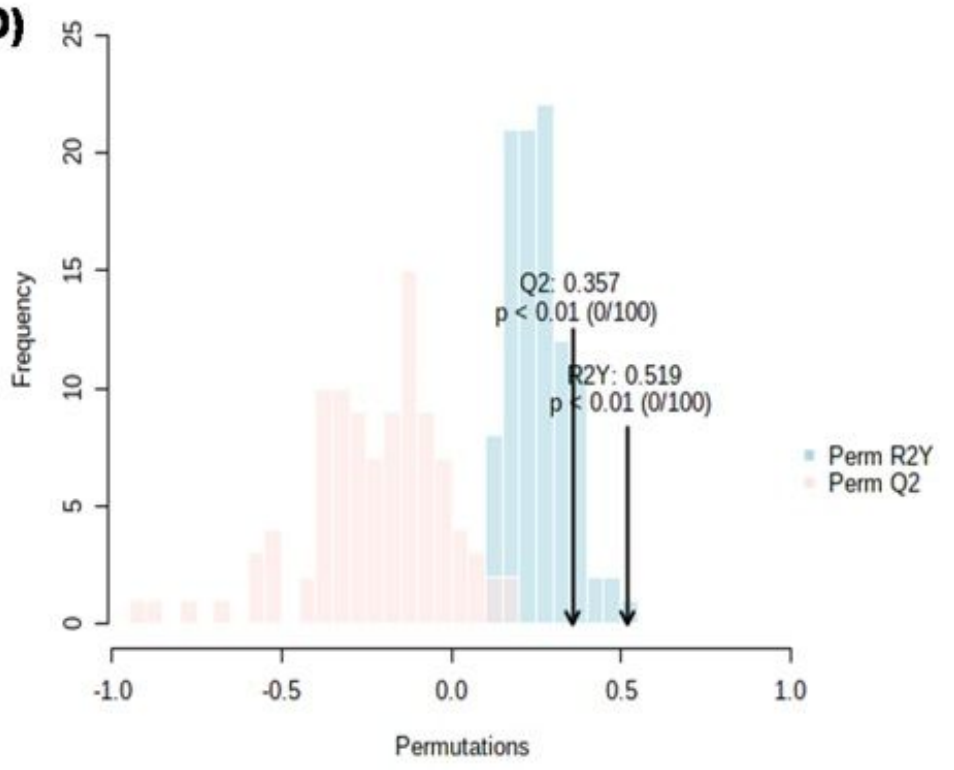

\section{Figure 1}

Results of multivariate analysis able to distinguish between the serum $1 \mathrm{H}-\mathrm{NMR}$ metabolic profiles of patients with sepsis and healthy controls - (A) PCA scores plot for discrimination showing PC1 (41\%) and PC2 (13.3) values, (B) The OPLS-DA score plot showing clear discrimination two groups, (C) VIP scores for 14 metabolites with highest contribution of to the separation of the present study groups and (D) Validation of OPL-DA by permutation test $(\mathrm{Q} 2=0.38$ and $\mathrm{R} 2 \mathrm{Y}=0.53)$ performed with 100 random permutations test. 


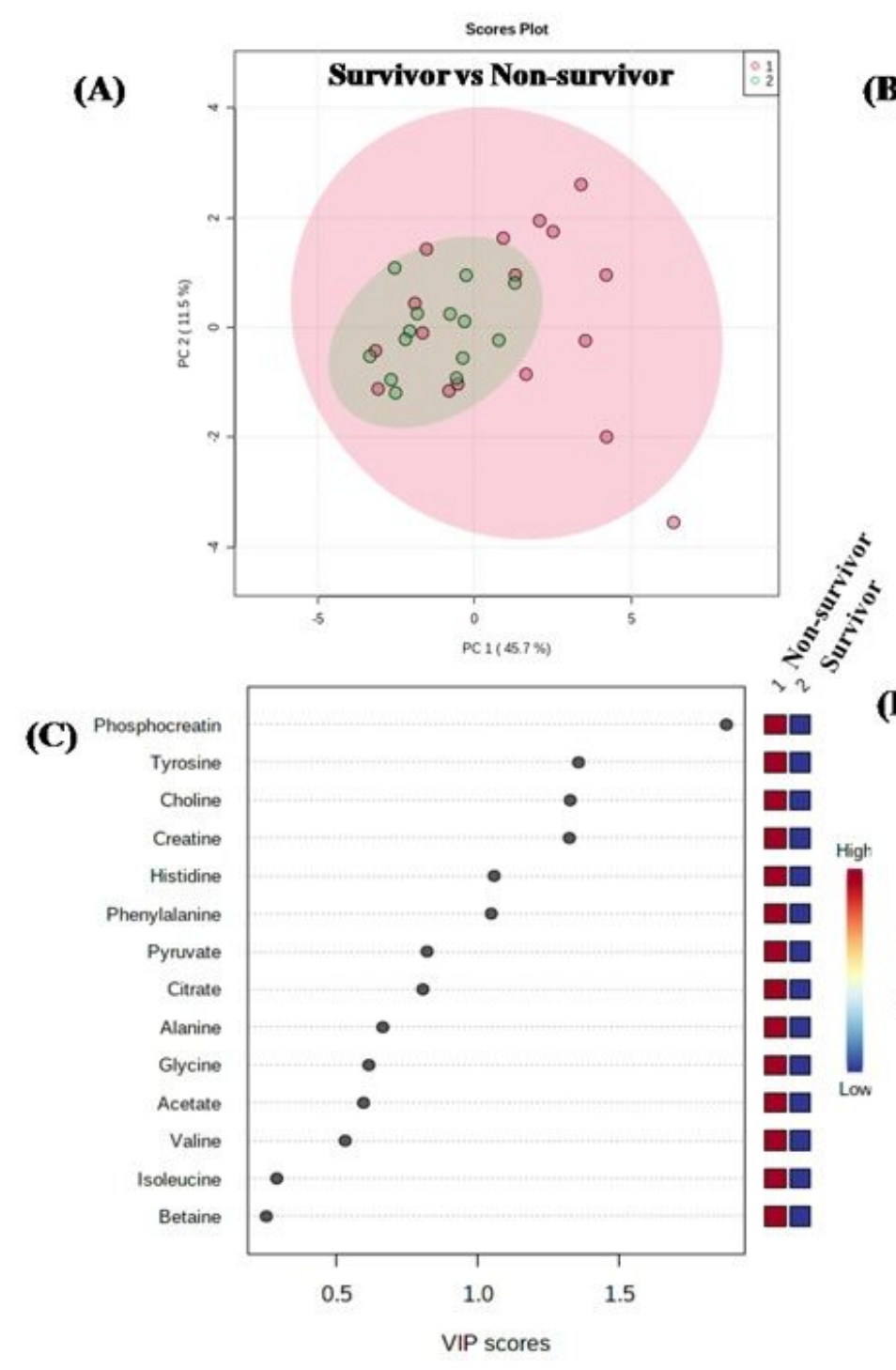

(B)
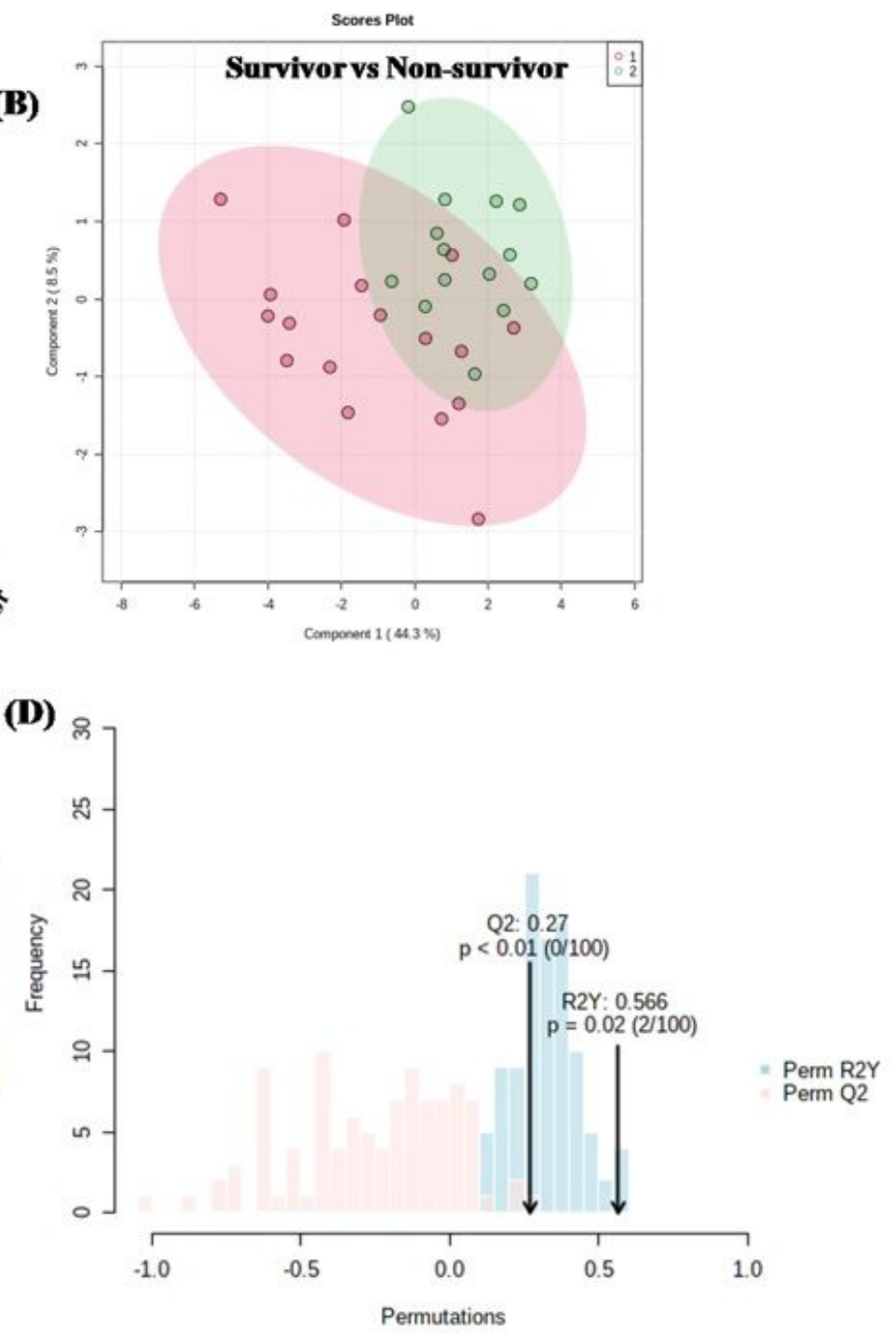

Figure 2

Results of multivariate analysis able to distinguish between the serum 1H-NMR metabolic profiles of patients with sepsis survivor and non-survivor- (A) PCA scores plot for discrimination showing PC1 (45.0\%) and PC2 (10.4\%) values, (B) The OPLS-DA score plot showing clear discrimination two groups, (C) VIP scores for 14 metabolites with highest contribution of to the separation of the present study groups and (D) Validation of OPL-DA by permutation $(\mathrm{Q} 2=0.435$ and $\mathrm{R} 2 \mathrm{Y}=0.669)$ test performed with 100 random permutations test 


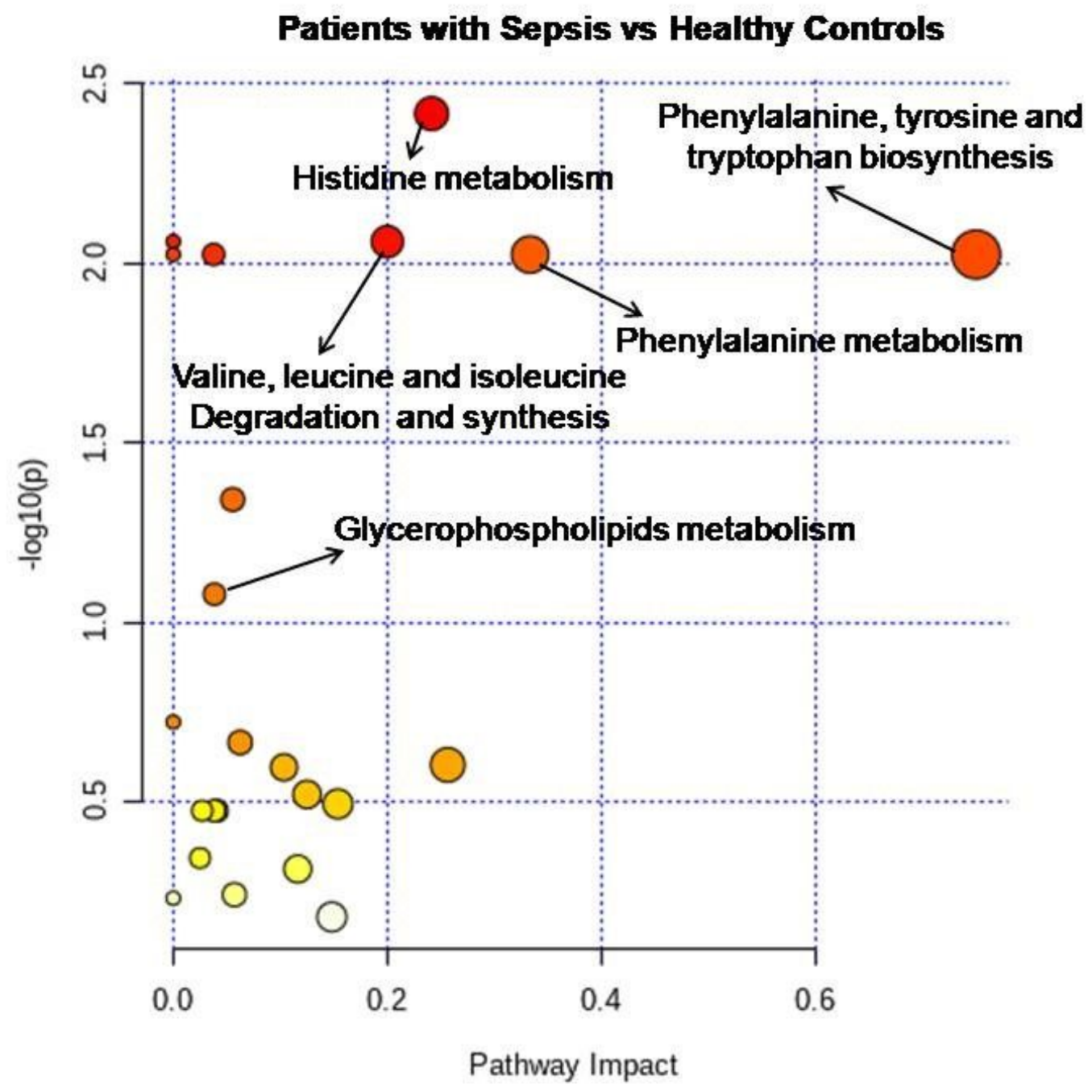

Figure 3

The altered metabolic pathways using the concentrations of metabolites from serum samples in the pathway analysis using MetaboAnalyst 4.0 software to reveal the most relevant pathways related to patients with sepsis vs. healthy controls. 

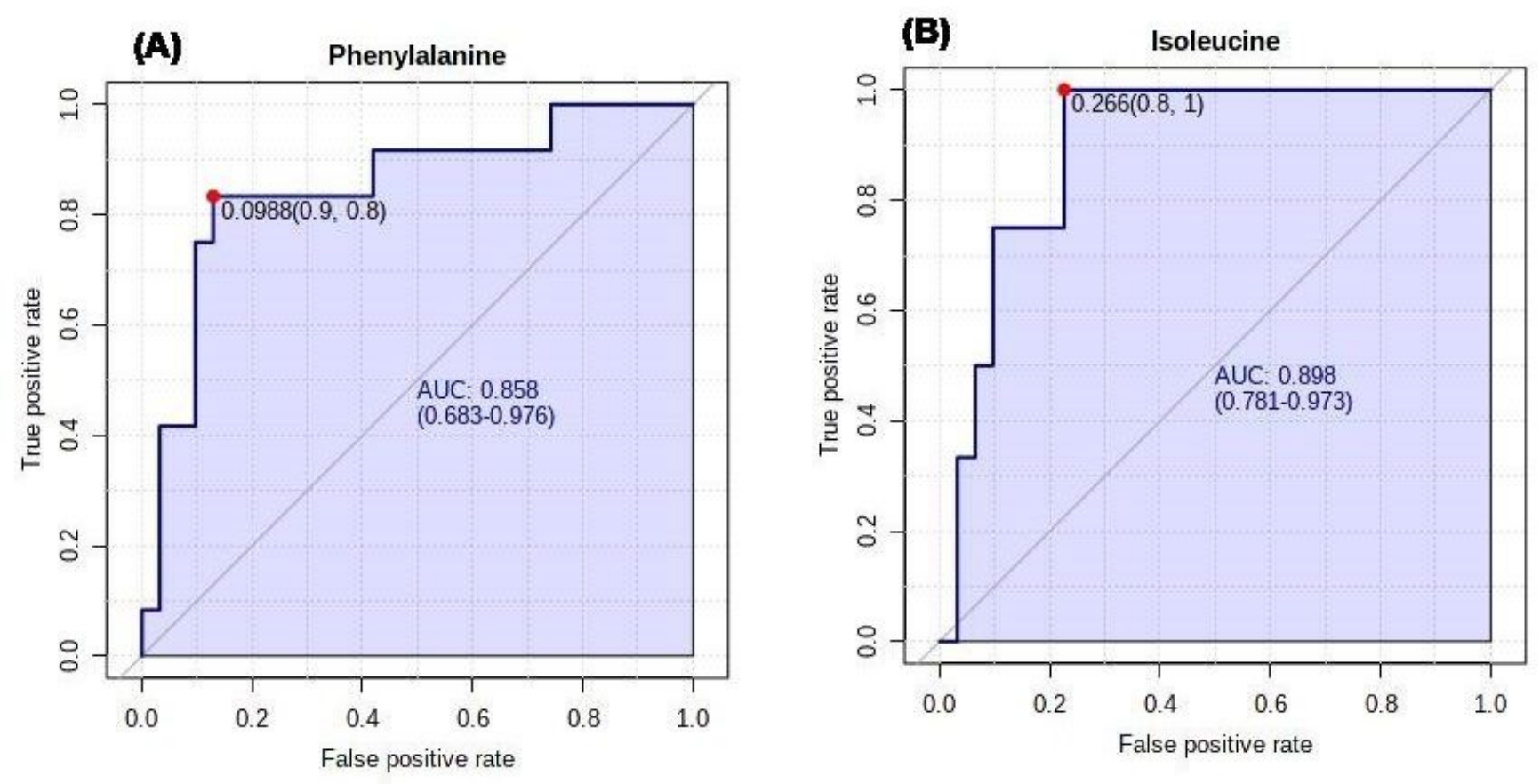

\section{Figure 4}

ROC curve analysis for individual serum metabolites (biomarker/s). The univariate ROC curves analysis for phenylalanine (A) and isoleucine (B) in serum with AUC values and 95\% IC of AUC (in brackets). Red dots refer to the optimal cutoffs for which specificity and sensitivity are given in brackets. 


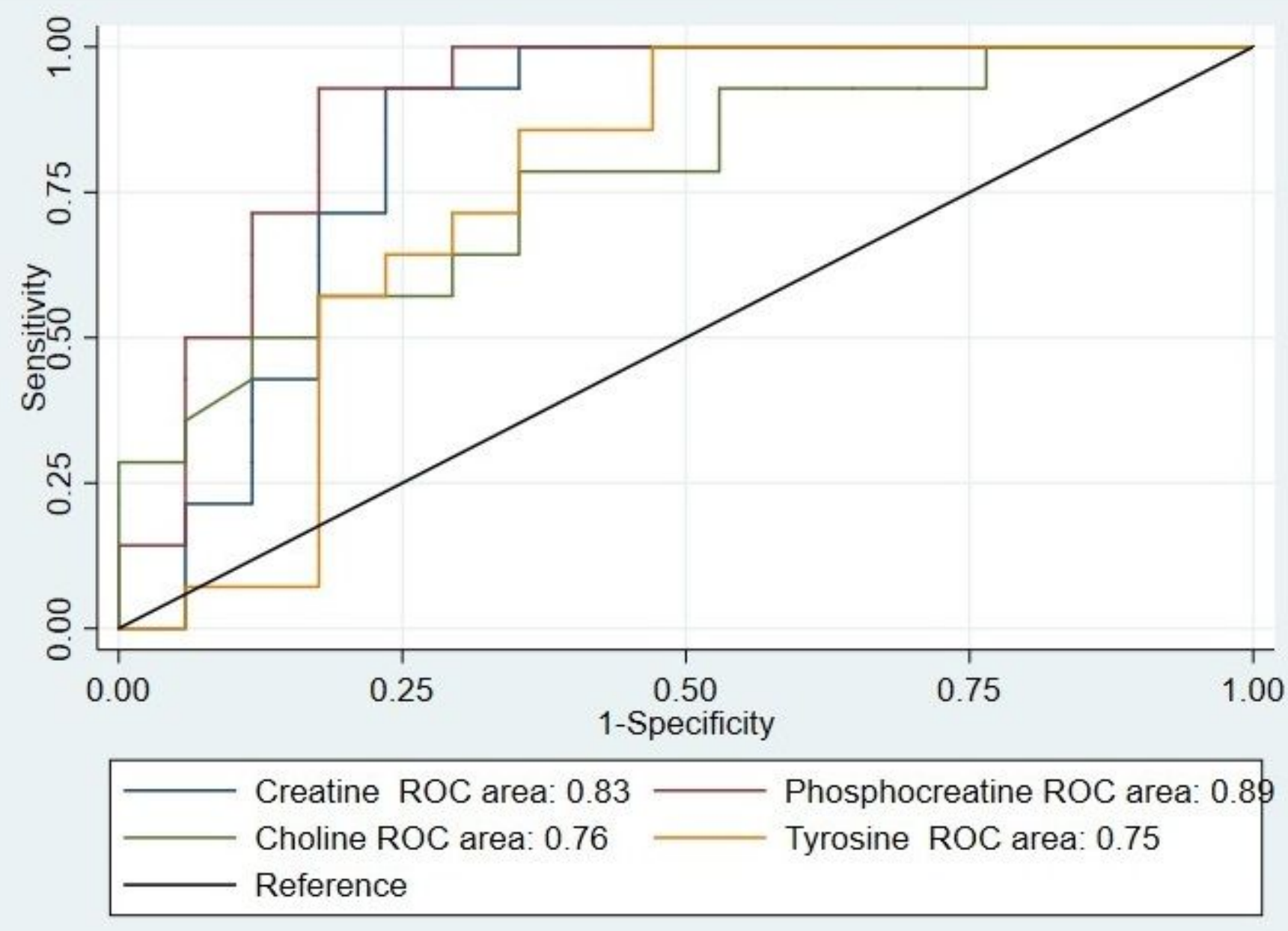

Figure 5

Multivariate Receptor Operator Curve (ROC) curves obtained from serum sample patients with sepsis nonsurvivors vs survivors. 